\title{
The Incidence of Disseminated Intravascular Coagulation in Dogs with Malignant Tumor
}

\author{
Haruhiko MARUYAMA $^{1)}$, Teruhisa MIURA ${ }^{1)}$, Manabu SAKAI ${ }^{1)}$, Hiroshi KOIE $^{1)}$, Yoshiki YAMAYA ${ }^{1)}$, \\ Hisashi SHIBUYA ${ }^{2)}$, Tsuneo SATO ${ }^{2)}$, Toshihiro WATARI ${ }^{1) *}$, Akira TAKEUCHI ${ }^{1)}$, Mikihiko TOKURIKI ${ }^{1)}$ and \\ Atsuhiko HASEGAWA ${ }^{3)}$ \\ ${ }^{1)}$ Laboratories of Comprehensive Veterinary Clinical Studies, ${ }^{2)}$ Veterinary Pathology and ${ }^{3)}$ Pathobiology, Department of Veterinary \\ Medicine, Nihon University, 1866 Kameino, Fujisawa, Kanagawa 252-8510, Japan
}

(Received 6 August 2003/Accepted 25 December 2003)

ABSTRACT. The incidence of DIC in 208 dogs with a malignant tumor was evaluated. The incidence of DIC was $9.6 \%$ in dogs with a malignant tumor which was a solid tumor in all. In $164 \mathrm{dogs}$ with a malignant solid tumor, the incidence of DIC was $12.2 \%$. The incidence of DIC in dogs with hemangiosarcoma, mammary gland carcinoma and adenocarcinoma of the lung was significantly higher than that in dogs with other malignant tumors. These results suggested that special care in looking for DIC should be taken in dogs with a malignant solid tumor.

KEY WORDS: canine, DIC, malignancy.

J. Vet. Med. Sci. 66(5): 573-575, 2004

Disseminated intravascular coagulation (DIC) is a complex syndrome characterized by excessive thrombin and plasmin formation, causing the consumption of platelets, coagulation factors and inhibitors, and secondary hyperfibrinolysis. Therefore, DIC shows a diffuse microthrombosis and bleeding tendency.

DIC was frequently diagnosed in dogs with an underlying disease such as malignant tumor, pancreatitis, sepsis, immune-mediated hemolytic anemia, hepatitis, heat stroke and trauma $[1,2]$. Among these various underlying diseases, malignant tumor was reported to be the commonest cause of DIC in dogs [2].

There are some reports concerning abnormal hemostasis or the incidence of DIC in several tumor diseases of dogs [3, $4,9,11]$, but the incidence of DIC remains unclear in malignant tumor cases.

This paper deals with the incidence of DIC in dogs with a malignant tumor.

Malignant tumor was diagnosed by histopathological or cytological examination in 208 dogs (164 dogs with malignant solid tumor and 44 dogs with malignant hematopoietic tumor) referred to the Animal Medical Center of Nihon University from May 1999 to February 2003. The coagulation examinations performed were prothrombin time (PT), activated partial thromboplastin time (APTT) and plasma fibrinogen concentration in 208 dogs, platelet count and plasma antithrombin III (AT III) activity in 205 dogs and serum fibrinogen/fibrin degradation products (FDP) in 153 dogs.

The platelet count was measured with an automatic blood cell counter (Nihon Kohden, Tokyo, Japan). PT, APTT and

\footnotetext{
* Correspondence to: Watari, T., Laboratory of Comprehensive Veterinary Clinical Studies, Department of Veterinary Medicine, Nihon University, 1866 Kameino, Fujisawa, Kanagawa 2528510, Japan.
}

the plasma fibrinogen concentration was measured with a semi-automated mechanical clot detection system (Heinrich Amelung GmbH, Lemgo, Germany) and PT reagent (Dade Behring, Illinois, U.S.A.), APTT reagent (Dade Behring, Illinois, U.S.A.) and thrombin reagent (Dade Behring, Illinois, U.S.A.), respectively. Serum FDP was measured with latex agglutination reagent (Teikoku Hormone Manufacturing Co., Tokyo, Japan). Plasma AT III activity was measured with synthetic substrate (Dade Behring, Illinois, U.S.A.). A human pool plasma was used to prepare the standard plasma ATIII activity curve.

DIC was diagnosed by four or more abnormal findings among the following: low platelet count $\left(<150 \times 10^{3} / \mu l\right)$, prolonged PT ( $>8.5 \mathrm{sec})$, prolonged APTT ( $>18.0 \mathrm{sec})$, low plasma fibrinogen concentration $(<200 \mathrm{mg} / \mathrm{d} l)$, low plasma AT III activity $(<90 \%)$ and high serum FDP $(\geq 10 \mu \mathrm{g} / \mathrm{ml})$. DIC was also diagnosed by three or more of the above, when FDP, AT III or a platelet count was not performed.

Fisher's exact test was used to compare the groups with and without DIC. A $p$ value of $<0.05$ indicated statistical significance.

A diagnosis of DIC was made in 20 of 208 dogs with a malignant tumor $(9.6 \%)$ (Table 1). All cases of DIC were dogs with a malignant solid tumor, and none with a malignant hematopoietic tumor which included lymphoma (38 cases), acute myeloid leukemia ( 2 cases), malignant histiocytosis ( 2 cases), acute lymphoblastic leukemia (1 case) and chronic lymphoblastic leukemia (1 case). In contrast to human cases in which DIC occurs frequently, DIC appears to be uncommon in dogs with a malignant hematopoietic tumor [8].

The incidence of DIC was $12.2 \%$ in 164 dogs with a malignant solid tumor, and that in human cases with a malignant solid tumor recently reported was $6.8 \%$ [10] and $10.9 \%$ [7]. Two reasons for the higher incidence of DIC in 
Table 1. The number of dogs with abnormal findings on coagulation examinations

\begin{tabular}{lcccc}
\hline $\begin{array}{c}\text { The number of abnormal findings on } \\
\text { coagulation examinations }\end{array}$ & $\begin{array}{c}\text { Malignant } \\
\text { solid tumors } \\
\mathrm{n}=164\end{array}$ & $\begin{array}{c}\text { Malignant } \\
\text { hematopoietic tumors } \\
\mathrm{n}=44\end{array}$ & $\begin{array}{c}\text { Total } \\
\mathrm{n}=208\end{array}$ \\
\hline DIC cases & 6 & $2^{*}$ & 0 & $2^{*}$ \\
& 5 & $8^{*}$ & 0 & $8^{*}$ \\
& 4 & $10^{*}$ & 0 & $10^{*}$ \\
\hline Non-DIC cases & 3 & $4^{*}$ & $2^{*}$ & $6^{*}$ \\
& 2 & 16 & 13 & 29 \\
& 1 & 49 & 13 & 62 \\
& 0 & 75 & 16 & 91 \\
\hline
\end{tabular}

*Checked by 6 kinds of coagulation examination.

Table 2. The incidence of DIC in dogs with a malignant tumor

\begin{tabular}{lccc}
\hline \multicolumn{1}{c}{ Tumor } & $\begin{array}{c}\text { DIC cases* } \\
\mathrm{n}(\%)\end{array}$ & $\begin{array}{c}\text { Non-DIC cases** } \\
\mathrm{n}(\%)\end{array}$ & $p$ value \\
\hline Hemangiosarcoma & $7(35)$ & $8(4.3)$ & $<0.001$ \\
Mammary gland carcinoma & $4(20)$ & $10(5.3)$ & $<0.04$ \\
Adenocarcinoma of the lung & $2(10)$ & $2(1.1)$ & $<0.05$ \\
Nasal adenocarcinoma & $2(10)$ & $16(8.5)$ & 0.69 \\
Squamous cell carcinoma & $1(5)$ & $15(8.0)$ & 1 \\
Osteosarcoma & $1(5)$ & $7(3.7)$ & 0.56 \\
Gastric carcinoma & $1(5)$ & $2(1.1)$ & 0.26 \\
Granulosa cell tumor & $1(5)$ & $1(0.5)$ & 0.18 \\
Adrenal cortical carcinoma & $1(5)$ & $1(0.5)$ & 0.18 \\
Others & $0(0)$ & $126(67.0)$ & - \\
\hline
\end{tabular}

$* 20$ dogs. $* * 188$ dogs.

Table 3. The results of coagulation examinations in dogs with a malignant tumor

\begin{tabular}{lccc}
\hline & Cases examined & DIC cases & Non-DIC cases \\
\hline Platelet $\left(\times 10^{3} / \mu l\right)^{*}$ & & & \\
$\quad$ Median & 358.5 & 121.0 & 369.0 \\
$\quad$ Mean & 350.2 & 140.3 & 373.0 \\
$\quad$ Range & $4-1010$ & $47-452$ & $4-1010$ \\
$\quad$ Dogs with decreased platelet count $(\%)$ & $36(17.6)$ & $14(38.9)$ & $22(61.1)$ \\
AT III $(\%)^{*}$ & & & \\
$\quad$ Median & 102.0 & 75.0 & 104.0 \\
Mean & 100.7 & 70.9 & 103.3 \\
$\quad$ Range & $26-169$ & $26-102$ & $49-169$ \\
Dogs with decreased AT III $(\%)^{*}$ & $65(31.7)$ & $16(24.6)$ & $49(75.4)$ \\
Dogs with prolonged PT** $(\%)$ & $33(15.9)$ & $15(45.5)$ & $18(54.5)$ \\
Dogs with prolonged APTT** $(\%)$ & $23(11.1)$ & $16(69.6)$ & $7(30.4)$ \\
Dogs with decreased fibrionogen** $(\%)$ & $43(20.7)$ & $16(37.2)$ & $27(62.8)$ \\
Dogs with high FDP*** $(\%)$ & $30(19.6)$ & $15(50.0)$ & $15(50.0)$ \\
\hline AT & &
\end{tabular}

AT III: antithrombin III, PT: prothrombin time, APTT: activated partial thromboplastin time, FDP: fibrinogen/fibrin degradation products. * Performed on 205 dogs. ** Performed on 208 dogs. *** Performed on 153 dogs.

the dog cases were suspected. The first one is that activities of some coagulation factors, factor V, VII, VIII, IX, XI and XII in dogs might be much higher than those in humans, readily causing DIC in dogs [6]. The second is that DIC in our study was diagnosed depending only on laboratory findings of activation of the clotting and fibrinolytic systems, and DIC in human cases was detected both by laboratory and clinical findings including thrombosis and hemorrhage.

Twenty dogs with DIC included 7 dogs with hemangiosarcoma, 4 dogs with mammary gland carcinoma, 2 dogs each with adenocarcinoma of the lung and nasal adenocarcinoma, 1 dog each with squamous cell carcinoma, osteosarcoma, gastric carcinoma, granulose cell tumor and adrenal cortical carcinoma. The incidence of DIC in dogs with hemangiosarcoma, mammary gland carcinoma, adenocarcinoma of the lung, nasal adenocarcinoma, squamous cell carcinoma, osteosarcoma, gastric carcinoma, granulose cell tumor and adrenal cortical carcinoma was $46.7 \%, 28.5 \%, 50.0 \%$, $11.1 \%, 6.3 \%, 12.5 \%, 33.3 \%, 50.0 \%$ and $50.0 \%$, respectively. The incidence of DIC was significantly higher in 
hemangiosarcoma $(p<0.001)$, mammary gland carcinoma $(p<0.04)$ and adenocarcinoma of the lung $(p<0.05)$ than that in the other malignant tumors (Table 2 ). These results indicated that special care should be taken in looking for coagulation abnormalities and DIC in dogs with hemangiosarcoma, mammary gland carcinoma, or adenocarcinoma of the lung. And care should also be taken care with dogs with other malignant tumors to look for coagulation abnormalities as well as DIC, since $56.3 \%$ of the dogs with a malignant tumor had abnormal coagulation findings in this study, including the dogs with DIC (Table 1) and $83 \%$ of dogs with cancer were reported to have some abnormal coagulation [5].

In 208 dogs with a malignant tumor, a low platelet count, prolonged PT, prolonged APTT, a low plasma fibrinogen concentration, low plasma AT III activity and high serum FDP was detected in 36, 33, 23, 43, 65 and 30 dogs, respectively (Table 3 ). Furthermore, among DIC cases, a low platelet count was detected in $14 \mathrm{dogs}(38.3 \%)$, prolonged PT in 15 dogs $(45.5 \%)$, prolonged APTT in 16 dogs (69.6\%), a low plasma fibrinogen concentration in 16 dogs (37.2\%), low plasma AT III activity in 16 dogs (24.6\%) and high serum FDP in 15 dogs $(50.0 \%)$. Therefore, when APTT is prolonged, DIC would be most suspected in dogs with a malignant tumor.

This study revealed that the incidence of DIC was $9.6 \%$ in dogs with a malignant tumor which was a solid tumor in all. In 164 dogs with a malignant solid tumor, the incidence of DIC was $12.2 \%$. The incidence of DIC in dogs with hemangiosarcoma, mammary gland carcinoma and adenocarcinoma of the lung was significantly higher than that in dogs with other malignant tumors.

\section{REFERENCES}

1. Carr, A.P., Panciera, D.L. and Kidd, L. 2002. J. Vet. Intern. Med. 16: 504-509.

2. Feldman, B. F., Madewell, B. R. and O'Neill, S. 1981. J. Am. Vet. Med. Assoc. 179: 151-154.

3. Hammer, A. S., Couto, C. G., Swardson, C. and Getzy, D. 1991. J. Vet. Intern. Med. 5: 11-14.

4. Hargis, A. M. and Feldman, B. F. 1991. J. Am. Vet. Med. Assoc. 198: 891-894.

5. Madewall, B. R., Feldman, B. F. and O'Neill, S. 1980. Thromb. Haemost. 44: 35-38.

6. Mischke, R. and Nolte, I. 2000. pp. 519-525. In: Schalm's Veterinary Hematology, 5th ed. (Feldman, B.F., Zinkl, J.G. and Jain, N.C. eds.), Lippincott Williams and Wilkins, Baltimore.

7. Okajima, K., Sakamoto, Y. and Uchiba, M. 2000. Am. J. Hematol. 65: 215-222.

8. O'Keefe, D. A. and Couto, C. G. 1988. Vet. Clin. North. Am. Small. Anim. Pract. 18: 157-168.

9. O'Keefe, D. A., Couto, C. G., Burke-Schwartz, C. and Jacobs, R. M. 1987. J. Vet. Intern. Med. 1: 75-80.

10. Sallah, S., Wan, J. Y., Nguyen, N.P., Hanrahan, L. R. and Sigounas, G. 2001. Thromb. Haemost. 86: 828-833.

11. Stockhaus, C., Kohn, B., Rudolph, R., Brunnberg, L. and Giger, U. 1999. J. Small Anim. Pract. 40: 326-331. 